\title{
A Comparison of Template Matching Registration Methods for Polarimetric Imagery
}

\author{
Daniel A. LeMaster \\ Air Force Institute of Technology \\ 2950 Hobson Way \\ Wright Patterson AFB, Ohio 45433 \\ 937-623-0895 \\ daniel.lemaster@afit.edu
}

\begin{abstract}
Automatic registration between channels in a passive polarimetric imaging system can be frustrated by naturally occurring intensity variations due to target polarization content. In this paper, three image registration algorithms are tested using polarimetric data in various stages of translational misregistration. These algorithms, known collectively as template matching methods, are cross-correlation, maximum mutual information, and phase correlation. Test data are derived from laboratory and HST Faint Object Camera (FOC) polarimetric imagery. Mutual information registration is shown to be robust against channel intensity inversions at a cost of significantly increased processing time. Conversely, cross-correlation provides the most consistent performance in low signal-tonoise conditions. ${ }^{12}$
\end{abstract}

\section{TABLE OF CONTENTS}

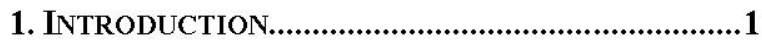

2. PolaRimetric IMAGE REgistration ...................2

3. REGISTRATION METHODS .....................................2

4. CoMPARISONS USING LABORATORY DATA ..........4

5. Comparisons Using HubBle Data......................6

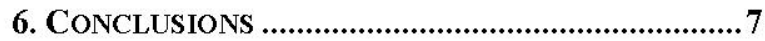

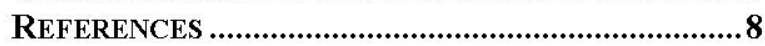

ACKNOWLEDGEMENTS ............................................9

BIOGRAPHY ..............................................................9

\section{INTRODUCTION}

Polarimetric Imaging (PI) is a form of remote sensing in which an object or event is characterized in terms of the polarization state of its reflected radiation. Though less common than panchromatic, multispectral, and radar imaging, passive polarimetric imaging has been applied successfully to a number of remote sensing problems. Astronomers have used PI to characterize the surface of planets and their atmosphere, to determine the surface properties of comets and asteroids, and to investigate the structure of galaxies $[1,2,3]$. Additionally, PI has been applied to earth surface characterization problems such as hydrology and oceanography as well as inventory and species identification problems in forestry and agriculture $[3,4]$. The military applications of polarimetric imagery

\section{1}

${ }^{1}$ U.S. Government work not protected by U.S. copyright.

${ }^{2}$ IEEEAC paper \#1082, Version 2, Updated December 1, 2007 include targeting, situational awareness and reconnaissance.

Imaging polarimeters for remote sensing can generally be placed into one of three categories: multi-channel, rotating polarizer, or micropolarizer array. A multi-channel polarimeter consists of two or more fixed polarizing elements through which the target scene is imaged simultaneously on individual detector arrays or sequentially (via a filter wheel) on a single detector array. Specific examples of each of these types of polarimeter are discussed in sections 5 and 6 . In the rotating polarizer case, the polarization image is formed by sampling the scene intensity at regular intervals as a polarizer is mechanically rotated. Finally, micropolarizer systems produce polarization imagery by sampling multiple fixed polarization states at the detector level and interpolating the result.

Both multi-channel and rotating polarizer imaging systems are prone to translational and rotational registration errors. As discussed in section 2, registration of multiple polarization channels is complicated by channel-to-channel intensity inversions and variations that are caused by highly polarized objects and regions in the target scene. An analogy can be made between registration of polarization imagery and multi-modal or multispectral imagery.

Adequate registration of polarization imagery requires one of two approaches: (a) manual selection of points or regions that are similar in each channel or (b) finding an automatic registration algorithm that is robust against polarization diversity. In many civilian and military remote sensing applications, manual registration is infeasible due to processing time constraints. Consequently, there is a need to identify or develop robust automatic registration methods.

Though a significant amount of work has been done on registering multispectral and multi-modal images (recent examples include $[6,7]$ ), relatively little work has been done on the specific problem of registering polarimetric imagery. Wang approached the problem of polarimetric image registration using wavelet transforms [8] and Persons [9] did the same using a Fourier phase technique similar to the phase correlation method presented in the following section. In both cases, these authors tested their algorithms against a fixed set of data to determine if a high level of subpixel 
registration could be achieved ${ }^{3}$. This paper is intended to compliment these previous works by identifying stressing polarimetric imaging registration scenarios to determine the conditions for total algorithm failure.

\section{Polarimetric Image Registration}

Many of the previously mentioned remote sensing applications of polarization imagery require estimation of the target polarization state at each pixel in the image. In passive collection applications, the polarization state of the underlying scene is most commonly described by the Stokes vector representation [10]. The Stokes vector consists of the four Stokes parameters:

$$
\mathrm{S}=\left[\begin{array}{llll}
\mathrm{S}_{0} & \mathrm{~S}_{1} & \mathrm{~S}_{2} & \mathrm{~S}_{3}
\end{array}\right]^{\mathrm{T}}
$$

For now, it is sufficient to state that:

- $\mathrm{S}_{0}$ is the total radiation intensity

- $\mathrm{S}_{1}$ and $\mathrm{S}_{2}$ together describe the orientation of the polarization ellipse

- $\mathrm{S}_{3}$ and $\mathrm{S}_{0}$ together describe its ellipticity

Plane (i.e. linear) polarization is more likely to occur in nature than elliptical polarization [11]. Consequently, $S_{3}$ is often assumed to be negligible. Without $S_{3}$, the Stokes parameters can be placed in a more familiar context: the angle of polarization, $\alpha$, and degree of linear polarization, $\mathrm{P}$ :

$$
\begin{gathered}
\alpha=\frac{1}{2} \tan ^{-1} \frac{S_{2}}{S_{1}} \quad-\frac{\pi}{2} \leq \alpha \leq \frac{\pi}{2} \\
P=\frac{\sqrt{S_{1}^{2}+S_{2}^{2}}}{S_{0}} \quad 0 \leq P \leq 1
\end{gathered}
$$

Simply stated, $\alpha$ describes the preferred orientation of the electric field vector and $\mathrm{P}$ describes the extent to which the radiation is polarized. Often, $\mathrm{P}$ is expressed as a percentage rather than a decimal.

The context of the misregistration problem can now be defined in terms of the relationship between the measured pixel intensities, $\mathrm{I}$, and the target Stokes parameters. This relationship for an $\mathrm{N}$ channel polarization imager is given by:

$$
\left[\begin{array}{c}
\mathrm{I}_{1} \\
\mathrm{I}_{2} \\
\vdots \\
\mathrm{I}_{\mathrm{N}}
\end{array}\right]=\left[\begin{array}{ccc}
\boldsymbol{\tau}_{1} & \boldsymbol{\tau}_{1} \cos 2 \theta_{1} & \boldsymbol{\tau}_{1} \sin 2 \theta_{1} \\
\boldsymbol{\tau}_{2} & \boldsymbol{\tau}_{2} \cos 2 \theta_{2} & \boldsymbol{\tau}_{2} \sin 2 \theta_{2} \\
\vdots & \vdots & \vdots \\
\boldsymbol{\tau}_{\mathrm{N}} & \boldsymbol{\tau}_{\mathrm{N}} \cos 2 \theta_{\mathrm{N}} & \boldsymbol{\tau}_{\mathrm{N}} \sin 2 \theta_{\mathrm{N}}
\end{array}\right]\left[\begin{array}{c}
\mathrm{S}_{0} \\
\mathrm{~S}_{1} \\
\mathrm{~S}_{2}
\end{array}\right]+\left[\begin{array}{c}
\varepsilon_{1} \\
\varepsilon_{2} \\
\vdots \\
\varepsilon_{\mathrm{N}}
\end{array}\right]
$$

where the $\tau$ terms represent total signal attenuation along the optical path, the angles $\theta$ represent the preferred polarization

\footnotetext{
3 both authors maintain that $1 / 10^{\text {th }}$ of a pixel registration accuracy is required to avoid unacceptable polarization artifacts
}

transmission $\operatorname{axes}^{4}$, and each $\varepsilon$ is a realization of the noise. The recovery of Stokes parameters from channel intensity measurements is therefore defined to be the (pseudo)inverse of (4).

Clearly, misregistration in $\mathrm{I}$, if left uncorrected, leads to erroneous estimates of the Stokes parameters. The registration methods presented in the following section attempt to find the correspondence between each channel in I via some similarity metric, however, variations in pixel-topixel Stokes parameters frustrate this effort.

To better explain the problem, consider the extreme case where the preferred polarization angle of the incident radiation is perpendicular to one of the polarimeter channels, in other words: $\alpha=\theta_{1}+\frac{\pi}{2}$. To do so, first recast $\mathrm{S}_{1}$ and $\mathrm{S}_{2}$ in terms of $\alpha$ and $\mathrm{P}$ :

$$
S=S_{0}\left[\begin{array}{c}
1 \\
P \cos 2 \alpha \\
P \sin 2 \alpha \\
0
\end{array}\right]
$$

Noting that:

$$
\begin{aligned}
\cos 2 \alpha & =\cos \left(2 \theta_{1}+\pi\right)=-\cos 2 \theta_{1} \\
\sin 2 \alpha & =\sin \left(2 \theta_{1}+\pi\right)=-\sin 2 \theta_{1}
\end{aligned}
$$

From equation (4) the measured intensity in the first channel would be:

$$
\begin{gathered}
I_{1}=\tau_{1} S_{0}-\tau_{1} S_{0} P \cos ^{2} 2 \theta_{1}-\tau_{1} S_{0} P \sin ^{2} 2 \theta_{1}+\varepsilon_{1} \\
=\tau_{1} S_{0}(1-P)+\varepsilon_{1}
\end{gathered}
$$

However channel 2 is oriented, it will not be parallel to channel 1. Thus, as the target becomes increasingly polarized (i.e. as $\mathrm{P} \rightarrow 1$ ), the signal in channel 1 decreases which forces a local intensity inversion between channels. When $\mathrm{P}=1$, the signal is lost in the noise.

Whereas the exact case presented above is unlikely to occur globally, regions of high polarization are bound to cause significant contrast variations between channels in many imaging polarimeter applications. As such, it is beneficial to identify registration algorithms that are robust against these contrast variations. To start this search, three existing registration techniques are presented in the following section for the purpose of testing their applicability to polarization imagery.

\section{REgistration Methods}

The three primary template matching registration methods (as defined by Zitova [15]) are presented in this section. There are many algorithm variations for each of these methods. In any case where there are alternatives, an effort

\footnotetext{
${ }^{4}$ off axis transmission is assumed to be negligible
} 
has been made here to adhere to the most common implementation.

Given two or more images, registration via template matching is achieved by selecting a region from a reference image (the template) and then finding the corresponding region in each of the remaining images (the window) by maximizing a similarity metric. This search is conducted under the assumption that the misregistration between images is purely translational. In the case of the multichannel polarimeter, choice of the reference image is arbitrary.

\section{Cross-Correlation}

Registration using cross-correlation is achieved by maximizing the correlation between two zero mean images. Mathematically, if $\mathrm{g}$ is the template and $\mathrm{f}$ is the window, then the normalized cross-correlation, $\mathrm{C}(\mathrm{u}, \mathrm{v})$, is given by [15]:

$$
=\frac{\mathrm{C}(\mathrm{u}, \mathrm{v})}{\sqrt{\sum_{\mathrm{m}} \sum_{\mathrm{n}} \mathrm{f}^{2}(\mathrm{~m}, \mathrm{n})} \sqrt{\sum_{\mathrm{m}} \sum_{\mathrm{n}} \mathrm{g}^{2}(\mathrm{~m}-\mathrm{u}, \mathrm{n}-\mathrm{v})}}
$$

where $(m, n)$ are the indices of the image and $(u, v)$ are the relative shifts between images. The translational shift required to register the images is the pair $(\mathrm{u}, \mathrm{v})$ that maximizes C. The numerator of (8) is the convolution of the template and window and can therefore be implemented using fast Fourier transforms. The normalizing effect of the denominator in (8) ensures that regions of high correlation but small overlap between the template and window are given sufficient weight when compared to regions with large overlap. As such, the cross correlation is bounded between -1 and 1 .

Cross-correlation registration of polarimetric imagery requires a number of special considerations. The raw polarization imagery will not be zero mean in general. Consequently, image means are subtracted as a preprocessing step. Furthermore, the cross-correlation coefficient is defined for two images. Since complete polarization state characterization requires at least three channels, cross-correlation registration must be implemented once for each pair of images.

Cross-correlation registration is most effective when the target and reference images are identical except for a translational shift and noise. In general, polarization imagery will contain content that does not meet this criterion. However, cross-correlation is a well known method that can be easily implemented and has been shown to be computationally faster than mutual information registration [13] and less susceptible to noise [12].

\section{Mutual Information}

The mutual information between images can also be used as a registration technique. In this case, mutual information is defined as [12]:

$$
\begin{aligned}
& \mathrm{M}(\mathrm{u}, \mathrm{v}) \\
& =\sum_{\mathrm{f}} \sum_{\mathrm{g}} \mathrm{p}_{\mathrm{FG}}(\mathrm{f}, \mathrm{g} \mid \mathrm{u}, \mathrm{v}) \log \left(\frac{\mathrm{p}_{\mathrm{FG}}(\mathrm{f}, \mathrm{g} \mid \mathrm{u}, \mathrm{v})}{\mathrm{p}_{\mathrm{F}}(\mathrm{f}) \mathrm{p}_{\mathrm{G}}(\mathrm{g})}\right)
\end{aligned}
$$

where $\mathrm{f}$ and $\mathrm{g}$ are allowed intensity values in the template image, $G$, and window image, $F \cdot p_{G}(g)$ and $p_{F}(f)$ are the probability mass functions (pmf) of the intensity values in $G$ and $F$, and $p_{F G}(f, g \mid u, v)$ is the joint pmf of the images given a relative shift $(\mathrm{u}, \mathrm{v})$ between them. The translational shift required to register the images is the pair $(\mathrm{u}, \mathrm{v})$ that maximizes $M$. Using this definition, mutual information is a distance measure of the statistical dependence between intensity values in $\mathrm{F}$ and $\mathrm{G}$. For the purposes of image registration, strong dependence (i.e. large $M$ ) between intensity values in $\mathrm{F}$ and $\mathrm{G}$ is assumed to be an indication of image registration. Said another way, mutual information is largest when the knowledge of a given intensity $f$ results in the least possible uncertainty in the value of $g$.

Equation (9) requires an additional stipulation for what occurs when $\mathrm{p}_{\mathrm{FG}}(\mathrm{f}, \mathrm{g} \mid \mathrm{u}, \mathrm{v})$ or $\mathrm{p}_{\mathrm{G}}(\mathrm{g}) \mathrm{p}_{\mathrm{F}}(\mathrm{f})$ equal zero. By convention [14], these contributions to the sum are assigned a value of 0 . The interpretation of this convention is that, when something occurs with zero probability, it will contain no information about the statistical dependence of $\mathrm{f}$ and $\mathrm{g}$.

The pmfs required to calculate $M$ are approximated from the images themselves. $p_{G}(g)$ and $p_{F}(f)$ are found from the normalized histogram of the window and template images. Likewise, the joint $\mathrm{pmf}$ is approximated to be the normalized two-dimensional histogram of $\mathrm{F}$ and $\mathrm{G}$.

Histogram bin size is a free parameter that can impact the reliability of the $\mathrm{MI}$ registration algorithm. A discussion of research that addresses this problem can be found in [15]. In this case, the method proposed by Scott [16] for determining optimum histogram bin width, $h$, is employed:

$$
\mathrm{h}=\frac{3.49 \sigma}{\sqrt[3]{n}}
$$

where $\sigma$ is the image standard deviation and $\mathrm{n}$ is the number of image samples. This bin width is most valid when the underlying data is Gaussian.

There is no consensus on the definition of mutual information for three or more random variables. Consequently, mutual information registration will be implemented once for each pair of images as in the crosscorrelation case. Since one set of pmf calculations are required at each translation pair $(\mathrm{u}, \mathrm{v})$, mutual information registration will be the most computationally expensive registration technique tested during the course of this research. 
The proposed mutual information technique is better suited than cross-correlation for the problem of registering polarimetric imagery because the data is represented by intensity probability distributions rather than the intensity values themselves. To explain this point, consider a (registered) region of high intensity in $\mathrm{F}$ and low intensity in G. This region would contribute a low cross-correlation score even though the images are registered. The joint pmf of $\mathrm{G}$ and $\mathrm{T}$ would show that this apparent intensity inversion occurs with high joint probability and would therefore receive a high mutual information score. This scenario is common in medical applications, for instance when registering Computed Tomography (CT) and Magnetic Resonance (MR) images of bone [17]. Likewise, inversions and other intensity mismatches are expected to occur in regions of high polarization content.

\section{Phase Correlation}

The final registration method addressed in this study is referred to as phase correlation [15]. In this case, the phase correlation objective function, $\mathrm{P}_{c}$, is given by:

$$
\mathrm{P}_{\mathrm{c}}=\mathcal{F}^{-1}\left\{\frac{\mathrm{GF}^{*}}{\left|\mathrm{GF}^{*}\right|}\right\}
$$

where $\mathcal{F}^{-1}$ is the inverse Fourier transform operator and $\mathrm{G}$ and $\mathrm{F}$ are the Fourier transforms of images $\mathrm{f}$ and $\mathrm{g}$. In this case, the translational shift required to register the images are the coordinates of the maximum value of $\mathrm{P}_{c}$.

Interpretation of the phase correlation method is straightforward. Consider the numerator on the inside of the inverse Fourier transform in equation (11): it is the spatial cross-power spectrum of the two images. Assume $f$ and $g$ are identical except for a misregistration of $u$ pixels in the $m$ direction and $\mathrm{v}$ pixels in $\mathrm{n}$. Mathematically, this scenario for the numerator is given by:

$$
\begin{aligned}
& G\left(\zeta_{m}, \zeta_{n}\right) F^{*}\left(\zeta_{m}, \zeta_{n}\right) \\
& =F\left(\zeta_{m}, \zeta_{n}\right) F^{*}\left(\zeta_{m}, \zeta_{n}\right) e^{-j 2 \pi\left(\zeta_{m} u+\zeta_{n} v\right)}
\end{aligned}
$$

where $\zeta_{m}$ and $\zeta_{n}$ are the spatial frequency indices in the Fourier domain. The normalizing effect of the denominator in (11) leaves only the exponential term in (12). The inverse Fourier transform of this exponential term is a delta function centered at $(\mathrm{u}, \mathrm{v})$, the relative shift between the images.

As discussed in the cross-correlation case, polarization imagery will not meet the assumption that $f$ and $g$ are identical but translated. Unlike cross-correlation, however, phase correlation is said to be more robust against image-toimage illumination variations [15]. Of the three methods tested here, phase correlation registration is, by far, the fastest.

\section{COMPARISONS USING LABORATORY DATA}

In this section, the cross-correlation, mutual information, and phase correlation registration algorithms are applied to data collected in the laboratory. By careful selection of the illumination source polarization, emphasis is placed on extreme cases where channel-to-channel registration is assumed to be difficult.

\section{Sensor Setup}

The sensor constructed for these tests consists of three separate imaging channels. All three channels share a common reducing optic and focal plane array. As shown in figure 1 , the target scene is imaged separately through each of the lenses in the channel array. Two of the elements in the channel array consist of a lens and polarizing filter oriented at 30 or -30 degrees with respect to the horizontal plane of the target scene. The third channel contains no polarizing elements. The limit of the intermediate images are determined by the channel stop, which is physically located at the target. These intermediate images are spatially separated from each other by the diameter of the lenses themselves. This composite of images is therefore made to fit onto the focal plane array via the reducing optic. Once digitized, the composite image is processed to form a single polarization image.

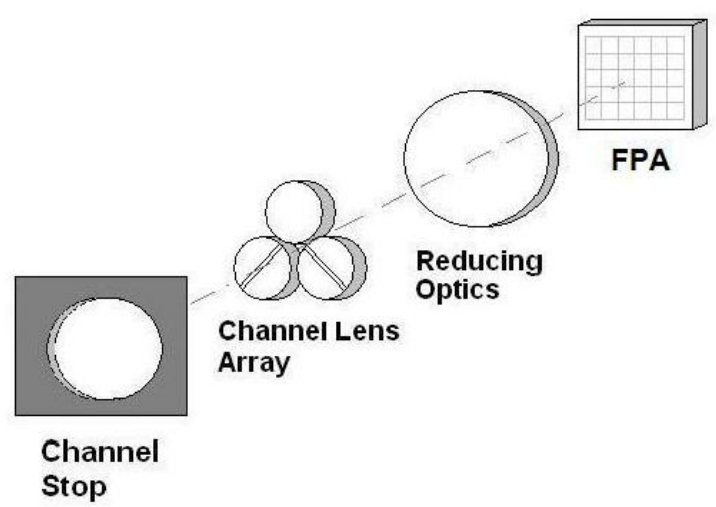

\section{Figure 1- Schematic Layout of the Laboratory Polarimeter}

By equation (4), the relationship between the raw channel data and target Stokes parameters is given by:

$$
\left[\begin{array}{l}
I_{1} \\
I_{2} \\
I_{3}
\end{array}\right]=\left[\begin{array}{ccc}
\tau_{1} & \tau_{1} \cos 2 \theta_{1} & \tau_{1} \sin 2 \theta_{1} \\
\tau_{2} & \tau_{2} \cos 2 \theta_{2} & \tau_{2} \sin 2 \theta_{2} \\
\tau_{3} & 0 & 0
\end{array}\right]\left[\begin{array}{l}
S_{0} \\
S_{1} \\
S_{2}
\end{array}\right]
$$

where $\tau_{1}, \tau_{2}$ and $\tau_{3}$ are predetermined calibration coefficients and $\theta_{1}, \theta_{2}$ are 30 and -30 degrees. Figure 2 contains an example of a raw 3-channel image. 


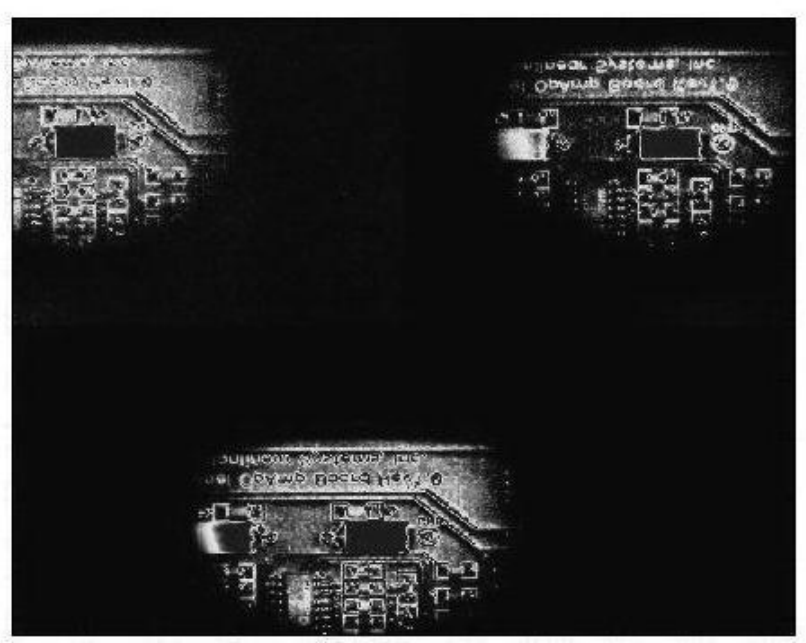

Figure 2- Laboratory Measurements from a Printed Circuit Board. The individual channels have been $2 \%$ stretched to enhance visual interpretability.

\section{Test Images}

Eight target scenes containing varying polarization content are collected using the previously described sensor. Scenes 1 through 3 contain a paper target with a printed design and aluminum foil. Regardless of the orientation of the illumination source, the paper tends to depolarize reflected light (average $\mathrm{P}<20 \%$ ). The foil, on the other hand, produces highly polarized reflections when illuminated by a polarized source. Scenes 4 and 5 contain the paper target, the foil and a linear polarizer oriented along image horizontal. Scenes 6 through 8 are of an integrated circuit board with generally unknown polarization characteristics and generally the lowest scene average signal to noise. With one exception, each scene is illuminated by polarized light of varying angle as determined by a polarizer at the source. An example of the circuit board image was shown previously in figure 2 . Figure 3 contains an example of the other targets types (from the unpolarized channel only).

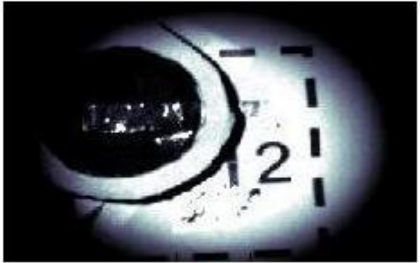

(a)

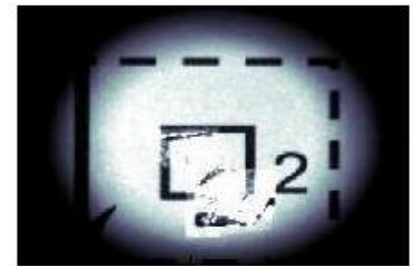

(b)
Figure 3 - Unpolarized Channel Views of the Remaining Laboratory Target Classes. (a) Paper, polarizer and foil. (b) Paper and Foil

The following table summarizes the characteristics of the 8 target scenes. The average degree of linear polarization (DOLP) is taken over the target region that intersects all three channel images. Likewise the scene average SNR is reported for each channel ${ }^{5}$ assuming a scene independent

\footnotetext{
5

5 While these averages are a us eful indicator, there is large radiometric and polarimetric contrast in any given image.
}

noise level of 7 counts (determined empirically).

Table 1 - Laboratory data synopsis

\begin{tabular}{l|ccccc}
\hline Scene & $\begin{array}{l}\text { Source } \\
\text { Polarization } \\
\text { Angle }\end{array}$ & $\begin{array}{l}\text { Average } \\
\text { DOLP }\end{array}$ & $\begin{array}{l}\text { SNR } \\
I_{30} \\
(d B)\end{array}$ & $\begin{array}{l}\text { SNR } \\
I_{30} \\
(d B)\end{array}$ & $\begin{array}{l}\text { SNR } \\
I \\
(d B)\end{array}$ \\
\hline 1 & $90^{\circ}$ & $45 \%$ & 13 & 12 & 24 \\
2 & $-30^{\circ}$ & $36 \%$ & 14 & 11 & 23 \\
3 & $-60^{\circ}$ & $38 \%$ & 14 & 10 & 24 \\
4 & $-60^{\circ}$ & $62 \%$ & 13 & 9 & 22 \\
5 & $-30^{\circ}$ & $58 \%$ & 14 & 12 & 23 \\
6 & $-30^{\circ}$ & $82 \%$ & 6 & 1 & 14 \\
7 & $90^{\circ}$ & $78 \%$ & 2 & 2 & 14 \\
8 & unpolarized & $49 \%$ & 8 & 7 & 18 \\
\hline
\end{tabular}

Experiment Execution

The test imagery was cropped and registered using easily identifiable (i.e. unpolarized) control points in each image. In this case, success or failure is determined by the methods' ability to predict that the polarization channels are already registered (or nearly so).

\section{Results}

The success or failure of the registration algorithms generally fell into two ranges: registration error on the order of a pixel or complete failure (errors $>30$ pixels). Table 2 summarizes these results. Misregistration of a single pixel should not be considered a failure because subpixel sampling differences between channels were not taken into account.

Table 2 - Registration of Laboratory Data: Test Results $\mathrm{X}$ represents complete failure, a numerical entry indicates the amount of registration error in whole pixels.

\begin{tabular}{l|lcl|lll|lll}
\hline & \multicolumn{2}{|l|}{\begin{tabular}{l}
\multicolumn{2}{l|}{ Cross- } \\
Correlation
\end{tabular}} & \multicolumn{2}{l|l}{$\begin{array}{l}\text { Mutual } \\
\text { Information }\end{array}$} & \multicolumn{2}{l}{$\begin{array}{l}\text { Phase } \\
\text { Correlation }\end{array}$} \\
\hline & $1-2$ & $1-3$ & $2-3$ & $1-2$ & $1-3$ & $2-3$ & $1-2$ & $1-3$ & $2-3$ \\
\hline 1 & & 1 & & & 1 & & & & \\
2 & & 1 & & 1 & & 1 & & & \\
3 & $\mathrm{X}$ & 1 & $\mathrm{X}$ & 2 & 1 & & $\mathrm{X}$ & & $\mathrm{X}$ \\
4 & $\mathrm{X}$ & & $\mathrm{X}$ & $\mathrm{X}$ & & & $\mathrm{X}$ & & \\
5 & $\mathrm{X}$ & & & & & & $\mathrm{X}$ & & \\
6 & & & & & & & & & \\
7 & & & & & & & & & \\
8 & & & & & & & & & \\
\hline
\end{tabular}

The failures of cross correlation and phase correlation in scenes 3,4 , and 5 are associated with large scale polarization diversity between channels. Figure 4 shows the intensity inversion that occurred between channels 1 and 2 in scene 3 . 


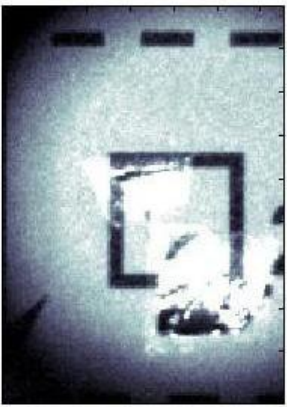

(a)

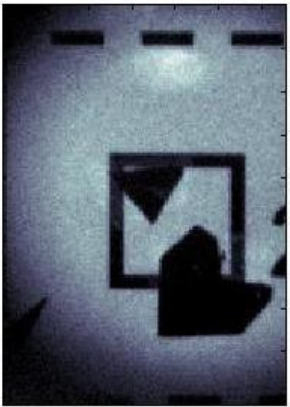

(b)
Figure 4-Intensity Inversion Due to Polarization Diversity. (a) Scene 3, channel 1. (b) Scene 3, channel 2.

Mutual information registration proved to be more robust against intensity inversions. This improved performance is gained at a substantial cost in computational efficiency. On average, the MI algorithm took 25.23 seconds to run per pair of images. Compare this result to 2.30 seconds for cross-correlation and 0.03 seconds for phase correlation.

Interestingly, the lowest SNR cases were not necessarily more prone to registration errors. That being said, registration between channels 1 and 3 or 2 and 3 was more likely to succeed than registration between channels 1 and 2 . Two possible (complimentary) explanations for this behavior are proposed: (a) channel 3 has a much higher SNR than either channels 1 or 2 in all cases and (b) being unencumbered by a polarizer, channel 3 may be more similar to channels 1 and 2 than they are to each other.

\section{Comparisons Using Hubble Data}

The registration of three channel polarization imagery from the Hubble Faint Object Camera (FOC) is addressed in this section. The FOC, which operated aboard the Hubble Space Telescope (HST) until early 2002, was a UV to near IR multispectral imaging platform for observations of astrophysical objects. Included amongst the FOC's various spectral filters were three $\mathrm{MgF}_{2}$ double Rochon prism polarization filters oriented at 0,60 , and 120 degrees (measured counterclockwise from the image $\mathrm{x}$ axis). These polarizers, alone or in combination with other filters, inherently introduce translational misregistration (see, for instance, [18] or [19]).

A brief literature search shows that astrophysical researchers have employed control point or correlation based registration (or both) as a preprocessing step to exploiting FOC data $[2,20]$. FOC images are included in this comparison because they provide an excellent source of polarization imagery that is unlike anything compared in the previous section.

Besides shifting the image, each FOC polarization filter introduces a different point spread function and unique spectral transmission characteristics. Also, the HST-FOC optical path introduces a slight polarization bias to the signal [19]. The differences in spectral transmission characteristics are largely emolliated via normalization in the cross-correlation and phase correlation cases and are a non-issue in the case of mutual information registration. The remaining issues are not addressed in this research.

The most significant difference between these images and the laboratory data are the large low signal regions dominated by Gaussian channel noise.

\section{Test Images}

The first two sets of test images are of galaxy NGC 1068, collected in February, 1995. Polarization in the scene is due to the scattering of UV radiation emitted from the obscured galactic nucleus. The average target degree of polarization is $20 \%$ with a peak of $65 \%$ as determined by Capetti [1]. An example image of NGC 1068 from each channel is shown in figure 5.
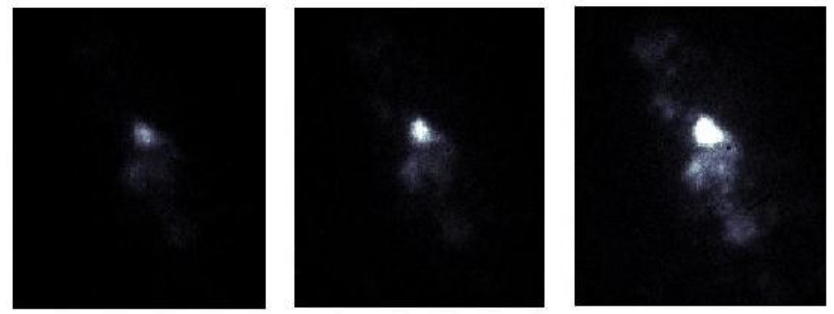

Figure 5- Hubble Space Telescope Polarization Imagery of Galaxy NGC 1068. From left to right: $\mathrm{I}_{0}, \mathrm{I}_{60}, \mathrm{I}_{120}$.

The next set of test images are of nebula $\mathrm{He} 3-1475$, collected in July, 1998. According to the corresponding HST collection abstract [21], this polarization imagery was to be used to partially determine the structure of the molecular torus close to the central star. It is not known if this effort was successful. Example images are shown in figure 6 .
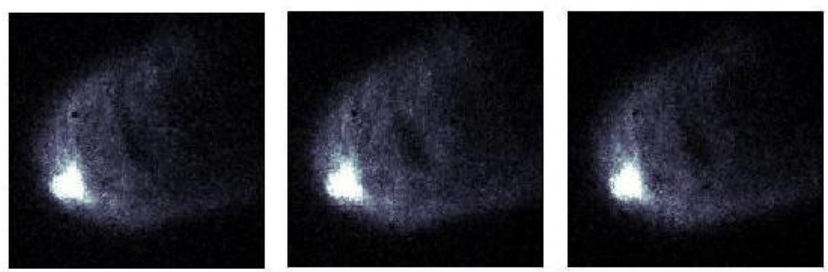

Figure 6 - Hubble Space Telescope Polarization Imagery of the Proto-Planetary Nebula He 3-1475. Only the central region is shown. From left to right: $\mathrm{I}_{0}, \mathrm{I}_{60}, \mathrm{I}_{120}$.

The final set of polarization images used in this test are of galaxy Markarian 3, collected in December, 1998. As in the NGC 1968 case, polarization in the scene is due to the scattering of UV radiation emitted from the obscured galactic nucleus. According to Kishimoto (et al.) [2], target degree of polarization starts in the $5-20 \%$ range near the 
center of the target and increases outward from there. The data itself is shown in figure 8 .
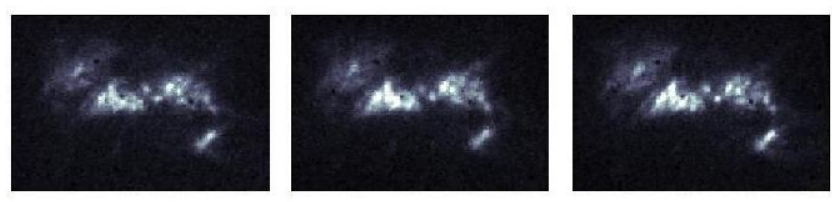

Figure 7 - Hubble Space Telescope Polarization Imagery of galaxy Markarian 3. From left to right: $I_{0}, I_{60}, I_{120}$.

\section{Experiment Execution}

Unlike the laboratory data cases, the precise amount of misregistration between these twelve pairs images is unknown. Though the inherent misregistration between FOC polarization channels is well documented, no registration data on the specific filter/polarizer combinations used in the measurements shown here is available. The predicted shifts between images are therefore compared across algorithms instead of to an absolute reference. Where one algorithm is an outlier, it is assumed to be in error. As before, registration errors are assumed to occur in whole pixel increments.

\section{Results}

Table 3 contains the predicted misregistration between each FOC polarization channel for each scene and each algorithm. Results are presented in terms of the required shift in the $\mathrm{x}$ and $\mathrm{y}$ directions ${ }^{6}$. Obvious outliers are highlighted in yellow.

\section{Table 3 - Predicted Misregistration Between} Astronomical Images

\begin{tabular}{|c|c|c|c|c|c|c|c|}
\hline \multirow[t]{2}{*}{ Dataset } & \multirow[t]{2}{*}{ Method } & \multicolumn{2}{|c|}{$I_{0} I_{60}$} & \multicolumn{2}{|c|}{$I_{0} I_{120}$} & \multicolumn{2}{|c|}{$I_{60} I_{120}$} \\
\hline & & $\mathrm{x}$ & $\mathrm{y}$ & $\mathrm{x}$ & $\mathrm{y}$ & $\mathrm{x}$ & $\mathrm{y}$ \\
\hline \multirow{4}{*}{$\begin{array}{l}\text { NGC } 1080 \\
\text { Set } 1\end{array}$} & & & & & & & \\
\hline & $\mathrm{CC}$ & -4 & -3 & 0 & 1 & 4 & 1 \\
\hline & $\mathrm{PC}$ & 0 & 0 & 0 & 0 & 0 & 1 \\
\hline & MI & -3 & -2 & 1 & -1 & 4 & 1 \\
\hline \multirow{4}{*}{$\begin{array}{l}\text { NGC } 1080 \\
\text { Set } 2\end{array}$} & & & & & & & \\
\hline & $\mathrm{CC}$ & -4 & -2 & 0 & 0 & 4 & 2 \\
\hline & $\mathrm{PC}$ & 0 & 0 & 0 & 0 & 4 & 2 \\
\hline & MI & -4 & -3 & 0 & -1 & 4 & 2 \\
\hline \multirow[t]{4}{*}{ He $3-1475$} & & & & & & & \\
\hline & $\mathrm{CC}$ & -2 & 1 & 0 & 0 & 2 & -1 \\
\hline & PC & -2 & 1 & 0 & 0 & 2 & -1 \\
\hline & MI & -3 & 0 & 0 & 0 & 2 & -1 \\
\hline \multirow[t]{4}{*}{ Mk 3} & & & & & & & \\
\hline & $\mathrm{CC}$ & -4 & 1 & 0 & 0 & 3 & 1 \\
\hline & PC & -4 & 0 & 0 & 0 & 4 & 1 \\
\hline & MI & -4 & 1 & 0 & 0 & 3 & 1 \\
\hline
\end{tabular}

\footnotetext{
7

${ }^{6}$ Following the Matlab convention, positive y is in the downward direction and positive $\mathrm{x}$ is a shift to the right
}

Under ideal conditions, the misregistration between any two pairs of images could be used to predict the misregistration between the third. As such, the general symmetry between the $I_{0}, I_{60}$ and $I_{60}, I_{120}$ columns is an indication of consistency throughout the measurements.

This is a very limited dataset considering the variety of possible astronomical targets and, as such, any general conclusions drawn here are going to have weak support. That being said, phase correlation would appear to provide the least consistent results in this case. Additionally, the data suggests that cross correlation provided the most consistent performance. Combining these two observations together, noise, rather than polarization content, would appear to be the limiting registration factor if these results can be reliably extrapolated.

\section{Conclusions}

Channel-to-channel variations in polarimetric imagery limit the effectiveness of automatic image registration algorithms. Three image registration methods are considered in this paper: cross-correlation, mutual information, and phase correlation. These algorithms are the principle constituents of a category of registration algorithms know as the areabased or template matching methods. Each method is tested against multi-channel laboratory and astrophysical polarimetric data to ascertain the conditions required for algorithm failure.

There are many more possible collection scenarios than there are test cases in this research. Consequently, the following conclusions are treated as precursors to a more general definitive statement about the registration of polarimetric imagery.

Within this scope, the following conclusions can be drawn from the laboratory data:

- Cross-correlation and phase correlation methods will fail in the presence of large scale intensity inversions between channels.

- In polarimetric imagery, the source of these intensity inversions is highly polarized scene content oriented orthogonally (or nearly so) to the preferred polarization angle of one or more sensor channels.

- Mutual information registration is more robust against intensity inversions at a cost of dramatically increased processing time.

- Phase correlation is computationally much faster than the other two methods.

Likewise, the following conclusions can be drawn from the astrophysical data: 
- Cross-correlation registration provides the most consistent performance, regardless of scene polarization.

- The performance of mutual information and phase correlation registration degrades appreciably in the presence of high noise levels.

The reversal in the effectiveness of the cross-correlation and mutual information methods between target regimes is hypothesized to be a function of the amount of polarization content in the scene and the average signal-to-noise level in the image.

\section{REFERENCES}

[1] Capetti, A., F. Macchetto, D. J. Axon, W. B. Sparks, and A. Boksenberg. Hubble Space Telescope Imaging Polarimetry of the Inner Nuclear Region of NGC 1068. Astrophysical Journal, 452:L87+, October 1995.

[2] Kishimoto, M., L. E. Kay, R. Antonucci, T. W. Hurt, R. D. Cohen, and J. H. Krolik. Ultraviolet Imaging Polarimetry of the Seyfert 2 Galaxy Markarian 3. Astrophysical Journal, 565:155-162, January 2002.

[3] Egan, Walter. Photometry and Polarization in Remote Sensing. Elsevier, New York, 1985.

[4] Whitehead, Victor and Kinsell Coulson. Remote Sensing in Polarized Light. NASA Conference Publication 3014. NASA Lyndon B. Johnson Space Center, NASA Scientific and Technical Information Division, November 3-5 1987.

[5] Collett, Edward. Polarized Light: Fundamentals and Applications. Marcel Dekker, Inc., New York, 1992.

[6] Yao, Jianchao and Kian Liong Goh. A Refined Algorithm for Multisensor Image Registration Based on Pixel Migration. IEEE Trans Image Process, 15(7):1839-1847, July 2006.

[7] Stone, Harold and Robert Wolpov. Blind CrossSpectral Image Registration Using Prefiltering and Fourier-Based Translation Detection. IEEE Trans Geoscience and Remote Sensing, 40(3):637-650, March 2002 .

[8] Wang, Xianbing, Shizhi Yang, Jinji Ma, and Yanli Qiao. Automated registration of polarimetric image using wavelet transform techniques. Volume 5832, 695702. SPIE, 2005.

[9] Persons, C. M., D. B. Chenault, M. W. Jones, K. D. Spradley, M. G. Gulley, and C. A. Farlow. Automated registration of polarimetric imagery using Fourier transform techniques. D. H. Goldstein and D. B.
Chenault (editors), Polarization Measurement, Analysis, and Applications V. Edited by Goldstein, Dennis H.; Chenault, David B. Proceedings of the SPIE, Volume 4819, pp. 107-117 (2002)., volume 4819 of Presented at the Society of Photo-Optical Instrumentation Engineers (SPIE) Conference, 107-117. September 2002.

[10] Egan, W. G. Polarization in Remote Sensing. Proc. SPIE Vol. 891, p. 2-9, Polarization Considerations for Optical Systems, volume 891 of Presented at the Society of Photo-Optical Instrumentation Engineers (SPIE) Conference, 2-9. 1988.

[11] Egan, W. G. Polarization in Remote Sensing. W. G. Egan (editor), Polarization and remote sensing; Proceedings of the Meeting, San Diego, CA, July 22, 23, 1992 (A93-30026 11-35), p. 2-48., volume 1747 of Presented at the Society of Photo-Optical Instrumentation Engineers (SPIE) Conference, 2-48. December 1992.

[12] Goshtasby, Ardeshir. 2-D and 3-D Image Registration for Medical, Remote Sensing, and Industrial Applications. John Wiley and Sons, Hoboken, NJ, 2005.

[13] A Flynn, G Boxer R B Pedley, A J Green and R H J Begent. A comparison of image registration techniques for the correlation of radio labelled antibody distribution with tumour morphology. Physics in Medicine and Biology, 44(7):N151-N159, 1999.

[14] MacKay, David J. C. Information Theory, Inference, and Learning Algorithms. Cambridge University Press, Cambridge, UK, 2003.

[15] Zitova, Barbara and Jan Flusser. Image registration methods: a survey. Image and Vision Computing, 21(11): 977-1000, October 2003.

[16] Scott, David. On Optimal and Data-Based Histograms. Biometrika, Vol. 66, No. 3 (Dec., 1979), pp. 605-610.

[17] Maes, F., A. Collignon, D. Vandermeulen, G. Marchal, and P. Suetens. Multimodality image registration by maximization of mutual information. Medical Imaging, IEEE Transactions on, 16(2):187-198, 1997.

[18] Hodge, P.E., Polarizer Throughputs and Image Shifts. Instrument Science Report FOC-089. 22 January 1996. www.stsci.edu/ftp/instrument_news/FOC/Foc_ist/foc _ sr089.ps

[19] The FOC (Faint Object Camera) Data Handbook http://www.stsci.edu/hst/HST overview/documents/dat ahandbook/

[20]Capetti, A.; Axon, D. J.; Macchetto, F.; Sparks, W. B.; 
B oksenberg, A. HST Imaging Polarimetry of NGC 1068. Astrophysical Journal v.446, p.155. 06/1995

[21] Harrington, J. Collimation of Astrophysical Jets: The Proto-Planetary Nebula He 3-1475. Hubble Space Telescope proposal 7285. Date??

\section{A CKNOWLEDGEMENTS}

Some of the data presented in this paper were obtained from the Multimission Archive at the Space Telescope Science Institute (MAST). STScI is operated by the Association of Universities for Research in Astronomy, Inc., under NASA contract NAS5-26555.

The opinions and views expressed by the author are not necessarily those of the Department of Defense or the United States Air Force.

\section{BIOGRAPHY}

Daniel A. LeMaster is an electrical engineering $P h D$

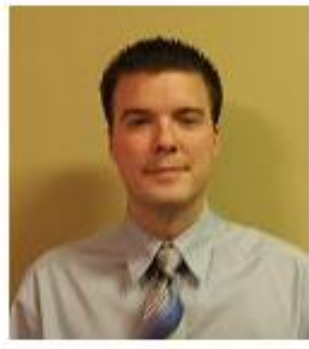
student at the Air Force institute of Technology (AFT) and a research scientist at the National Air and Space Intelligence Center (NASIC). He has a MS in Applied Physics from AFIT and a BSE in Engineering Physics from Wright State University. 